\title{
Harmonic analysis of fiber Bragg gratings written using apodized phase and amplitude masks
}

\section{Análisis armónico de las redas de Bragg de fibra grabadas utilizando máscaras de fase y de amplitud apodizadas}

\author{
Z. Jaroszewicz ${ }^{1,2, S^{*}}$, T.Osuch ${ }^{1,3}$ \\ 1. National Institute of Telecommunications, Szachowa 1, 04-894 Warsaw, Poland \\ 2. Institute of Applied Optics, ul. Kamionkowska 18, 03-805 Warsaw, Poland \\ 3. Institute of Electronic Systems, Warsaw University of Technology, Nowowiejska 15/19, 00-665 Warsaw, \\ Poland \\ (*) E-mail: mmtzjaroszewicz@post.pl S: SEDOPTICA member \\ Received: 30/12/2016 Accepted: 23/08/2017 \\ DOI: $10.7149 /$ OPA.50.3.49071
}

\begin{abstract}
:
In this paper theoretical research on harmonic response of apodized fiber Bragg gratings written using apodized phase and amplitude masks are presented. Two apodization techniques, namely variable phase step and variable fill factor are employed, and their influence on gratings' reflection efficiency of Bragg wavelength and its harmonic is extensively analyzed. Results show that using an appropriate apodization technique and profile power spectra of apodized fiber Bragg gratings can be widely tailored.
\end{abstract}

Key words: apodization, apodized phase and amplitude masks, fiber Bragg gratings, harmonic analysis

\section{RESUMEN:}

En este trabajo se presentan las investigaciones teóricas sobre la respuesta armónica de las redes de Bragg de fibra apodizadas grabadas utilizando máscaras de fase y de amplitud apodizadas. Se emplean dos técnicas de apodización, a saber, con escalón de fase variable y con factor de llenado variable, y se analiza ampliamente su influencia sobre la eficiencia de reflexión de las rejillas para la longitud de onda de Bragg y de su armónico. Los resultados muestran que el uso de una técnica de apodización apropiada y el perfil adecuado, pueden modificar ampliamente los espectros de potencia de las redes de Bragg de fibra apodizadas.

Palabras clave: Apodización, máscaras de fase y de amplitud apodizadas, redes de Bragg de fibra, análisis armónico

\section{REFERENCES AND LINKS / REFERENCIAS Y ENLACES}

[1] R. Kashyap, Fiber Bragg Gratings. New York, NY, USA: Academic (2010).

[2] T. Osuch and Z. Jaroszewicz, "Numerical analysis of apodized fiber Bragg grating formation using phase mask with variable diffraction efficiency," Opt. Commun. 284, 567-572 (2011). https://doi.org/10.1016/j.optcom.2010.09.063

[3] Z. Xiong, G. D. Peng, B. Wu, and P. L. Chu, "Effect of the zero-order diffraction of a phase mask on Bragg gratings," J. Lightwave Technol. 17, 2361-2365 (1999).

https://doi.org/10.1016/i.optcom.2010.09.063 
[4] J. D. Mills, C. W. J. Hilman, B. H. Lott, and S. Brocklesby, "Imaging of free space interference patterns used to manufacture fiber Bragg gratings," Appl. Opt. 39, 6128-6135 (2000). https://doi.org/10.1364/A0.39.006128

[5] T. Osuch and Z. Jaroszewicz, "Analysis of the Talbot effect in apodized diffractive optical elements," Photon. Lett. Poland 1, 190-192, (2009). https://doi.org/10.4302/plp.2009.4.16

[6] Z. Jaroszewicz, A. Kołodziejczyk, A. Kowalik, and R. Restrepo, "Determination of the step height of the binary phase grating from its Fresnel images," Optik 111, 207-210 (2001).

[7] F. J. Torcal-Milla, L. M. Sanchez-Brea, F. J. Salgado-Remacha, "Self-images location of amplitude/phase binary gratings," Appl. Opt. 48, 6252-6258 (2009). https://doi.org/10.1364/A0.48.006252

[8] T. Erdogan, "Fiber grating spectra, “J. Lightw. Technol. 15, 1277-1294 (1997). https://doi.org/10.1109/50.618322

[9] T. Osuch, K. Markowski, K. Jędrzejewski, "Numerical model of tapered fiber Bragg gratings for comprehensive analysis and optimization of their sensing and strain-induced tunable dispersion properties, “ Appl. Opt. 54, 5525-5533 (2015). https://doi.org/10.1364/A0.54.005525

[10] S. A. Wade, W. G. A. Brown, H. K. Bal, F. Sidiroglou, G. W. Baxter, S. F. Collins, "Effect of phase mask alignment on FBG spectra at harmonics of the Bragg wavelength," J. Opt. Soc. Am. A 29 1597-1605 (2012).

https://doi.org/10.1364/JOSAA.29.001597

\section{Introduction}

Fiber Bragg gratings (FBG) are one of the most rapidly developing and most applied passive fiber components. They owe their popularity to the wide possibilities of spectral characteristic formation both amplitude (reflexive) and phase (dispersive). One of the techniques used for creation of desired FBG characteristics is apodization. Its goal is minimization of the modulation amplitude of the grating's refractive index on its ends. Among various methods used for apodization implementation [1], the application of element with variable diffraction efficiency exhibits significant merits. Firstly, gratings obtained by use of apodized phase masks have constant effective refractive index. Thanks to that the undesired broadening of the reflectance characteristics for greater frequencies does not appear. Secondly, this method assures good repeatability, is relatively simple and does not require a UV laser of good coherence for the FBG exposure.

Classical (non-apodized) rectangular phase mask with a phase step of $\phi=\pi$ radians and fill ratio equal to 0.5 assures maximizing of first diffraction orders (on the value of approximately $40 \%$ ) and minimizing of zero and even diffraction orders. However, experience shows that even a small value of the zero [2,3] and higher than one $(m> \pm 1)$ [4] diffraction orders has an essential influence onto the distribution of the diffraction field behind the phase mask for the process of FBG exposure. Moreover, it was shown that non zero diffraction orders with indices $m=0$ and $m> \pm 1$ have also a significant influence onto the effectiveness of the grating formation for the wavelength corresponding to the harmonics of the basic Bragg wavelength $\lambda_{B}$, where $\lambda_{B}=2 \cdot n_{\text {eff }} \Lambda=n_{\text {eff }} \cdot \Lambda_{P M}, n_{\text {eff }}$ denominates the effective refraction index of the fiber and $\Lambda$ stands for the grating's period (or the period of the interference pattern created with the help of the phase mask having a period of $\Lambda_{\mathrm{PM}}$ ).

Diffraction efficiencies in the particular diffraction orders of the apodized gratings are even more diverse because of their variable diffraction efficiency. And so, in the case of apodization by the variable step height of the phase mask with constant fill factor $\alpha=0.5$ for the phase difference smaller than $\phi=\pi$, there appear non zero values in zero diffraction order and the remaining diffraction efficiencies of odd orders are varying as it is shown in Fig. 1a.

On the other hand, in the case of apodized phase masks with variable fill factor $\alpha$ (and constant height of the phase step corresponding to the phase difference $\phi=\pi$ ), for $\alpha<0.5$ there appear non zero values for both even as well as odd diffraction orders, what can be seen in Figs 1b and 1c. 
a)

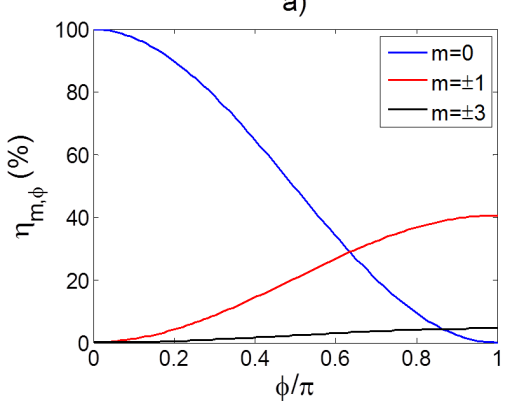

b)

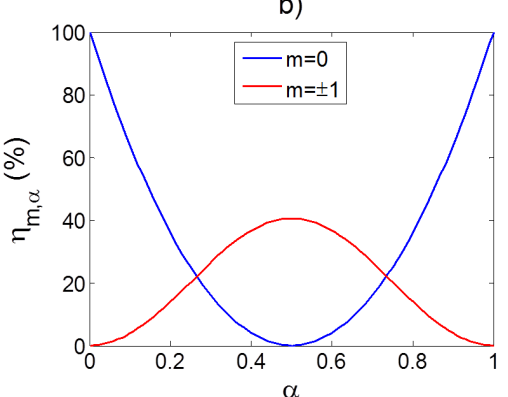

c)

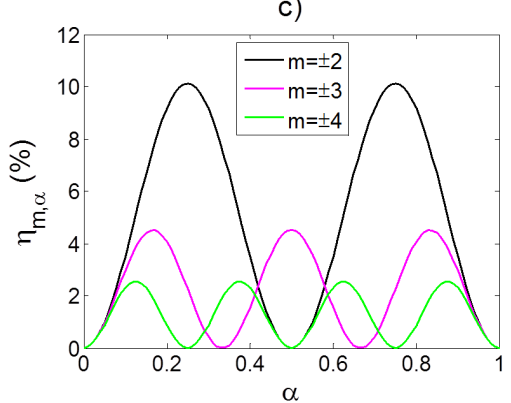

Fig. 1. Diffraction efficiency of phase masks with variable: a) phase step height, b), c) fill factor.

As a result the complex amplitude distribution behind the apodized phase masks are definitely more complicated than in the case of the uniform phase mask [2,5]. Hence, there can be expected a significant influence of apodization (higher diffraction orders) to the growth of Bragg resonance and harmonic reflections in apodized FBG.

In the present paper there will be shown an influence of apodization of phase/amplitude masks and different ways of variable diffraction efficiency implementation onto harmonic response of apodized FBGs. In chapter 2 the analysis method is presented, in chapter 3 the obtained results are shown, whereas chapter 4 contains summary of the work.

\section{Numerical model}

A method of harmonic response analysis of apodized fiber Bragg gratings is shown schematically in Fig.2. In this algorithm the diffraction field behind the phase/amplitude mask illuminated by a plane wave $\mathrm{E}(\mathrm{x}, \mathrm{z})$ is calculated as a superposition of amplitudes $A_{m}$ of all diffraction orders describing the transmittance of the analyzed mask. Then, the averaged field intensity $\mathrm{I}_{\mathrm{AV}}(\mathrm{x})$ within the core of optical fiber (where the FBG is formed) is derived. Finally, the harmonic response (i.e. power spectrum) $S\left(\lambda / \lambda_{B}\right)$ of the apodized FBG is obtained using the fast Fourier transform (FFT).

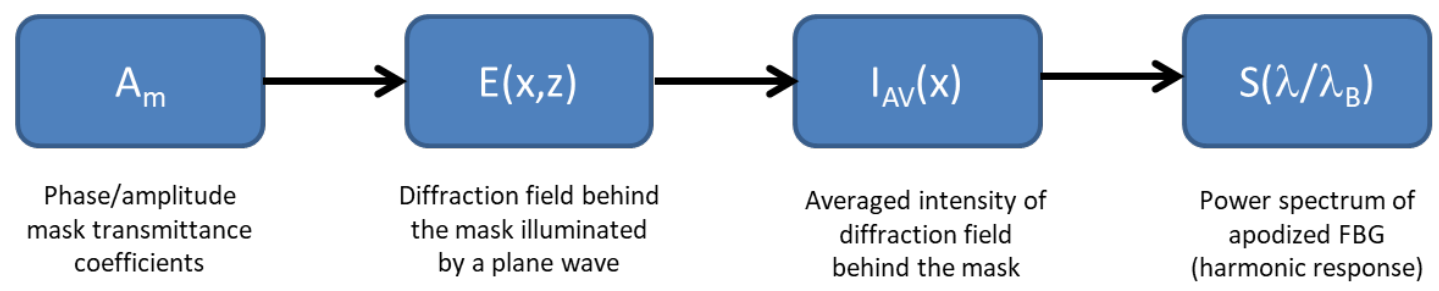

Fig. 2. Block diagram of the algorithm for analysis of the harmonic response of apodized FBGs.

Thus, the first step is the determination of the diffractive optical element's (i.e., phase mask) transmittance with the help of the electric field amplitudes $A_{m}$ of the m-th diffracted order of the phase mask section with a phase step height of $\phi$ (of fill factor $\alpha$ ).

From a technological point of view an ideal (continuous) apodization profile can be implemented in the phase mask as a finite number of different sections (with different height of the phase step or fill factor). Therefore, in the present analysis, it was assumed that the apodized phase mask is a symmetric structure consisting of $2 \mathrm{~N}-1$ sections (N - number of sections with different values of $\phi$ or $\alpha$ ). Gaussian profile of apodized phase mask is shown in Fig. 3a as a N-level stepped curve approximation.

In the case of the phase mask with variable phase step height the complex amplitude $A_{m}$ of m-th diffracted order of the phase mask section with the phase step of $\phi$ can be derived from the following formula [6]:

$$
A_{m}=\exp (-i \pi m) \exp \left(\frac{i \varphi}{2}\right) \operatorname{sinc}\left(\frac{m}{2}\right) \cos \left(\frac{\pi m-\varphi}{2}\right)
$$


a)

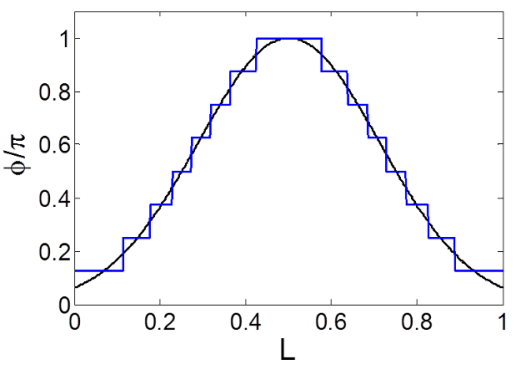

b)

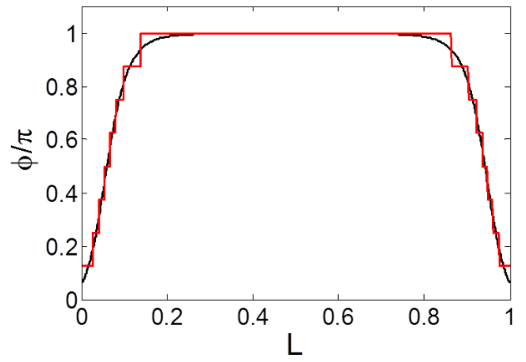

Fig. 3. Shape of the phase mask with a) Gaussian apodization and b) Tanh apodization and their approximation with 8-level stepped curve.

In turn, when a rectangular binary amplitude/phase grating having the duty cycle of $\alpha$ is illuminated by the plane wave with the unit amplitude, its complex amplitude of $\mathrm{m}$-th diffraction order can be expressed as [7]:

$$
A_{m}=\left\{\begin{array}{ccc}
b+\alpha \cdot[a \cdot \exp (-i \varphi)-b] & \text { for } & m=0 \\
\alpha \cdot[a \cdot \exp (-i \varphi)-b] \cdot \operatorname{sinc}(m \alpha) & \text { for } & m= \pm 1, \pm 2, \ldots
\end{array}\right.
$$

where $\mathrm{a}$ and $\mathrm{b}$ are the local transmittances of two different strips of the grating, $\phi$ stands for a phase step produced by the phase mask with a phase step height of $h=\lambda_{u v} \cdot \phi /\left[2 \pi\left(n_{u v}-1\right)\right]$, and $\lambda_{u v}$ is the wavelength of the UV laser used for FBG inscription, $\mathrm{n}_{\mathrm{uv}}$ denotes the refractive index of the phase mask substrate at $\lambda_{\text {uv }}$. For $\mathrm{a}=1$ and $\mathrm{b}=1$, pure phase mask is obtained.

Diffraction field $\mathrm{E}(\mathrm{x}, \mathrm{z})$ created at a distance $\mathrm{z}$ from the phase mask is described by the following formula [4]:

$$
E(x, z)=\sum_{m} A_{m} \exp (i m G x) \exp \left(i k_{m} z\right)
$$

where $\mathrm{k}_{\mathrm{m}}=\left(\mathrm{k}^{2}-\mathrm{m}^{2} \mathrm{G}^{2}\right)^{1 / 2}, \mathrm{G}=2 \pi / \Lambda_{\mathrm{PM}}, \mathrm{k}=2 \pi \mathrm{n}_{\mathrm{uv}} / \lambda_{\mathrm{uv}}, \mathrm{x}$ and $\mathrm{z}$ are axes perpendicular and parallel to the UV beam direction respectively, and $\mathrm{n}_{\mathrm{uv}}$ is refractive index of the medium behind the phase mask at writing wavelength $\lambda_{\text {uv. }}$ It means that the resultant field is a contribution of each diffraction order which coherently overlaps behind the phase mask. Corresponding intensity distribution can be expressed as $\mathrm{I}(\mathrm{x}, \mathrm{z})=|\mathrm{E}(\mathrm{x}, \mathrm{z})|^{2}$. In practice, due to the contribution of 0 -th and $m> \pm 1$ diffraction orders, the field distribution resulting mainly from \pm 1 order interference is no longer $\mathrm{z}$-independent. Thus, the periodicity of the fringe formed in a photosensitive fiber core during the FBG inscription can be described by an averaged intensity distribution $\mathrm{I}_{\mathrm{AV}}(\mathrm{x})$ behind the phase mask section:

$$
I_{A V}(x)=\int_{z}^{z+d} I(x, z) d z=\int_{z}^{z+d} E(x, z) \cdot E^{*}(x, z) d z,
$$

where $d$ is a fiber core diameter. When $I_{A V}(x)$ is analysed using FFT, the resultant spectrum consists of a series of peaks, which correspond to the Bragg wavelength and its harmonic components, which are formed in the FBG spectrum as a result of UV irradiation through the phase mask.

However, in the case of apodized structures such as phase masks with variable duty cycle or phase step, a piecewise-uniform approach can be used [8], where the whole non-uniform mask is divided into a finite number of $n=1,2, \ldots, N$ uniform sections (as in the case of apodized and/or tapered FBG analysis [9]). Each $n-$ th phase mask section is characterized by its normalized length $\Delta l_{n}=l_{n} / L$, and local duty cycle $\alpha_{n}$ or phase step $\phi_{\mathrm{n}}$ (where $\mathrm{l}_{\mathrm{n}}$ is absolute length of the $\mathrm{n}$-th section and $\mathrm{L}$ is the total length of the phase mask). Then, the total power spectrum $S\left(\lambda / \lambda_{B}\right)$ can be expressed as a weighted arithmetic mean of power $\operatorname{spectra} S_{n}\left(\lambda / \lambda_{B}\right)$ corresponding to the particular sections:

$$
S\left(\lambda / \lambda_{B}\right)=\sum_{n=1}^{N} S_{n}\left(\lambda / \lambda_{B}\right) \cdot \Delta l_{k}=\sum_{n=1}^{N}\left|F F T\left(I_{A V}^{n}(x)\right)\right| \cdot \Delta l_{n}
$$


where $I_{A V}^{n}(x)$ is an averaged intensity distribution within the fiber core corresponding to the $\mathrm{n}$-th section of an apodized phase mask.

\section{Results}

Considering that a phase mask with the period of $\Lambda_{\mathrm{PM}}=1 \mu \mathrm{m}$ is illuminated by the UV plane wave of the wavelength of $\lambda_{\mathrm{uv}}=244 \mathrm{~nm}$, only diffraction orders with numbers $m=0, \pm 1, \ldots, \pm 4$ are generated. The difference between core and cladding refractive indices at $\lambda_{\mathrm{uv}}=244 \mathrm{~nm}$ is small enough to assume that $\mathrm{n}_{\mathrm{co}}=\mathrm{n}_{\mathrm{cl}}=\mathrm{n}_{\mathrm{uv}}=1.5$. Fiber core diameter of $9 \mu \mathrm{m}$ was assumed.

\section{3.a. Influence of the apodization method on the harmonic content in apodized FBG spectrum}

In order to analyze the influence of the apodization manner on the Bragg wavelength and harmonics in apodized FBG power spectrum, the Gaussian profile was chosen, where the continuous apodization function was approximated by a step function of $\mathrm{N}=8$ levels. Thus, the analyzed phase masks were composed of $2 \mathrm{~N}$ 1=15 sections. Lengths of phase mask sections were chosen to approximate the apodization profile as best as possible. Two methods of apodization were considered:

a) By changing the phase step heights, where their values for different sections were equal to $\phi=\pi / 8$, $2 \pi / 8,3 \pi / 8, \ldots, \pi$, and the fill factor was constant for the whole mask and was equal to $\alpha=0.5$.

b) By changing the fill factor of the mask, where fill factor values of the subsequent sections were equal to $\alpha=0.0625,0.0125,0.1875,0.25, \ldots, 0.5$, and the phase step height was equal for the whole mask and amounted to $\phi=\pi$.

For comparison there were performed calculations for the non-apodized (uniform) phase mask given by $\alpha=0.5$ and $\phi=\pi$.

a)

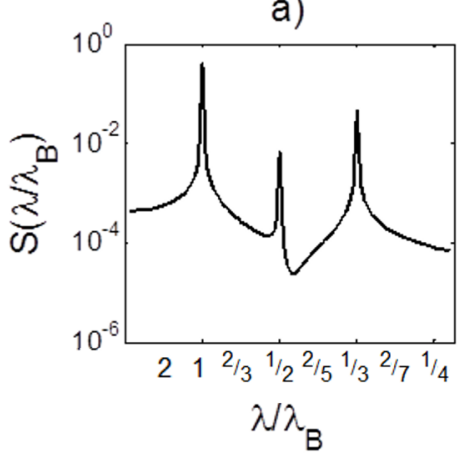

b)

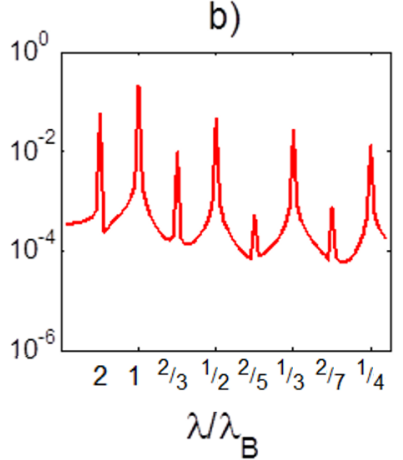

c)

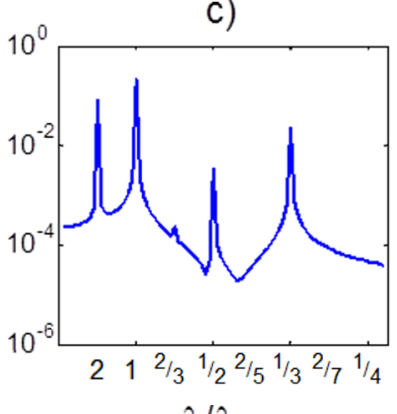

Fig. 4. Power spectrum of Bragg wavelength and harmonics components in apodized FBGs: a) non-apodized FBG, b) Gaussian apodized by varying fill factor, c) Gaussian apodized by varying phase step.

TABLE 1. Peak values of power spectra presented in Fig 4.

\begin{tabular}{|c|c|c|c|c|}
\hline \hline \multirow{2}{*}{$\Lambda / \Lambda_{\mathrm{PM}}$} & $\lambda$ & \multicolumn{3}{|c|}{$\mathrm{S}\left(\lambda / \lambda_{\mathrm{B}}\right)$} \\
\cline { 2 - 5 } & & Uniform & $\begin{array}{c}\text { Gaussian apodized } \\
\text { (variable } \alpha)\end{array}$ & $\begin{array}{c}\text { Gaussian apodized } \\
(\text { variable } \phi)\end{array}$ \\
\hline \hline 1 & $2 \lambda_{\mathrm{B}}$ & -- & $1,84 \mathrm{E}-02$ & $8,09 \mathrm{E}-02$ \\
$1 / 2$ & $\lambda_{\mathrm{B}}$ & $3,99 \mathrm{E}-01$ & $3,51 \mathrm{E}-01$ & $2,05 \mathrm{E}-01$ \\
$1 / 3$ & $2 \lambda_{\mathrm{B}} / 3$ & -- & $3,49 \mathrm{E}-03$ & $2,32 \mathrm{E}-04$ \\
$1 / 4$ & $\lambda_{\mathrm{B}} / 2$ & $6,64 \mathrm{E}-03$ & $1,70 \mathrm{E}-02$ & $3,36 \mathrm{E}-03$ \\
$1 / 5$ & $2 \lambda_{\mathrm{B}} / 5$ & -- & $2,26 \mathrm{E}-04$ & -- \\
$1 / 6$ & $\lambda_{\mathrm{B}} / 3$ & $4,49 \mathrm{E}-02$ & $3,88 \mathrm{E}-02$ & $2,31 \mathrm{E}-02$ \\
$1 / 7$ & $2 \lambda_{\mathrm{B}} / 7$ & -- & $3,29 \mathrm{E}-04$ & -- \\
$1 / 8$ & $\lambda_{\mathrm{B}} / 4$ & -- & $4,04 \mathrm{E}-03$ & -- \\
\hline \hline
\end{tabular}

As it can be seen, a significant difference between power spectra is a content of individual frequencies (Bragg's and harmonics). Only in the case of the phase mask apodized by variable fill factor (Fig. $4 \mathrm{~b}$ ), power spectrum contains all harmonics, i.e. $\Lambda / \Lambda_{\mathrm{PM}}=1 / \mathrm{p}$ (where $\left.\mathrm{p}=1,2, \ldots, 8\right)$. This is due to the fact that the complex 
amplitude distribution behind such masks is a result of interference of all non-zero diffraction orders, i.e., $\mathrm{m}=0$ and $\mathrm{m}= \pm 1, \ldots \pm 4$. In other words, all spatial frequencies, corresponding to the harmonic components $\Lambda / \Lambda_{\mathrm{PM}}=1 /(\mathrm{m}+\mathrm{n})$, result from the interference between $\mathrm{m}$-th and $\mathrm{n}$-th diffraction orders. Although, for some values of fill factor some orders are equal to zero (see Fig. 1c), but the power spectrum is composed of diffraction orders contributions coming from all sections. Spectral components $\Lambda=\Lambda_{\mathrm{PM}} / \mathrm{p}$ correspond to the Bragg wavelength peak at $\lambda_{B}(p=2)$ and the other peak reflection wavelengths $\lambda_{p}$ can be derived by the following formula [10]:

$$
\lambda_{p}=2 n_{\text {eff }} \Lambda=2 n_{\text {eff }} \Lambda_{P M} / p=2 \lambda_{B} / p
$$

where $\mathrm{n}_{\text {eff }}$ is the effective refractive index of the fiber core at $\lambda_{\mathrm{p}}$.

In the case of the phase mask apodized by the change of the phase step height as well as for a uniform phase mask some spectral components are lacking due to the zero diffraction efficiencies for even diffraction orders. In turn, components with wavelengths $2 \lambda_{B}$ and $2 \lambda_{B} / 3$, (absent in the case of FBG exposed by the uniform phase mask) are a result of interference of 1 and 0 as well as 0 and 3 diffraction orders accordingly. In turn, in the case of the uniform phase mask only 1 and 3 diffraction orders appear, hence spectral components present in Fig. $4 \mathrm{a}$ are the result of interference of orders +1 and $-1,1$ and 3 as well as +3 and 3.

Moreover, in all cases the highest value shows the peak corresponding to the basic Bragg wavelength $\lambda_{B}$ (as a product of beating of +1 and -1 diffraction orders).

Concluding, it can be stated that apodization with variable fill factor offers greater opportunities for shaping grating's reflectance for both the Bragg wavelength and its particular harmonics and that's because of all non-zero amplitudes in the power spectrum.

\section{3.b. Influence of the apodization function's shape onto the harmonic content of the apodized FBG spectrum}

In order to evaluate the influence of the apodization function's shape onto the content of harmonic frequencies of the power spectra there were compared results obtained for the uniform FBG with FBG apodized with Gaussian and Tanh functions (Fig. 5). It was assumed that the masks were apodized by variable fill factor.

a)

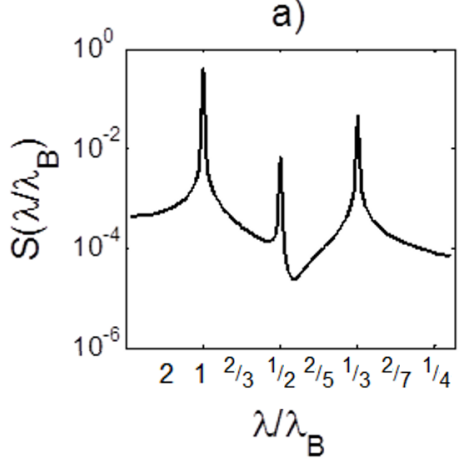

b)

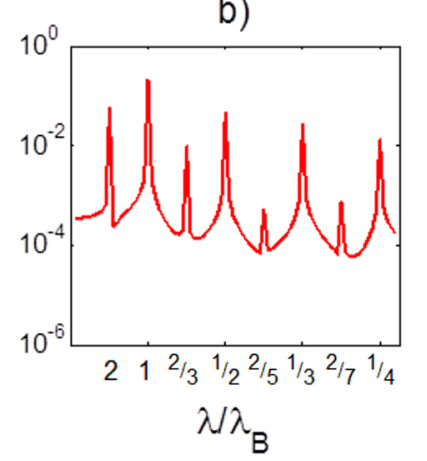

c)

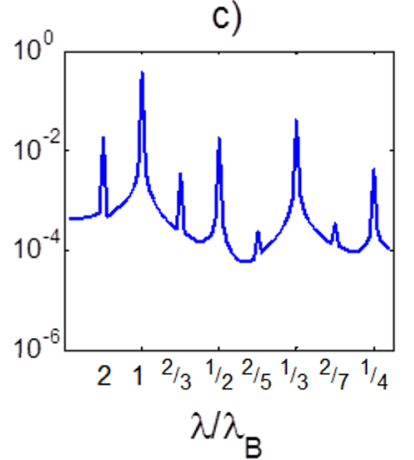

Fig. 5. Power spectra of FBG: a) uniform, b) with Gaussian apodization function c) with Tanh apodization function, obtained by varying the fill factor.

As it can be seen in the case of Tanh apodization, values of harmonic peaks $\lambda_{B}, \lambda_{B} / 2$ and $\lambda_{B} / 3$ are similar to those found in power spectrum of the uniform mask. Besides that, other peaks are smaller than in the case of Gaussian apodization. The reason for such a high amplitude is the shape of the Tanh apodization profile, which is more rectangular (as in the case of uniform FBG) than the Gaussian profile. Thus, a relatively long central section of the Tanh apodized FBG operates as a uniform Bragg grating, where Bragg resonance corresponding to the $\lambda_{B}, \lambda_{B} / 2$ and $\lambda_{B} / 3$ are more effectively reflected. Because of the same reason, an influence of the zero order onto harmonic corresponding to $2 \lambda_{B}$ is significantly smaller for the Tanh apodized grating in comparison with the Gaussian apodized one. Therefore, choosing appropriately the apodization function, the effectiveness of the reflectance for particular harmonics can be shaped to a certain extent. 


\section{3.c. Amplitude mask apodized with variable fill factor}

Substituting to eq. (2) $\phi=0$; complex amplitude of m-th diffraction order of the amplitude mask can be written as follows:

$$
A_{m}=\left\{\begin{array}{ccc}
b+\alpha \cdot(a-b) & \text { for } & m=0 \\
\alpha \cdot(a-b) \cdot \operatorname{sinc}(m \alpha) & \text { for } & m= \pm 1, \pm 2, \ldots
\end{array} .\right.
$$

Thus, the diffraction efficiency for particular diffraction orders of so defined amplitude mask (for a=1 and $b=0$ ) in function of the fill factor is shown in Fig. 6 .

a)

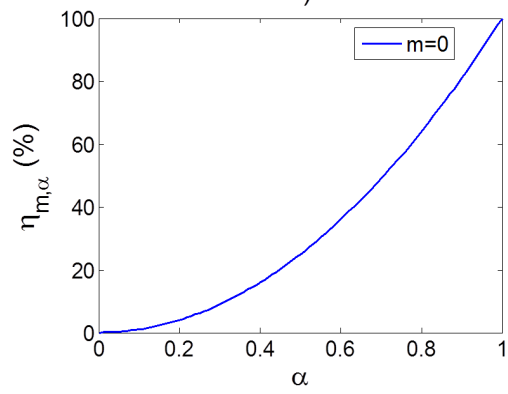

b)

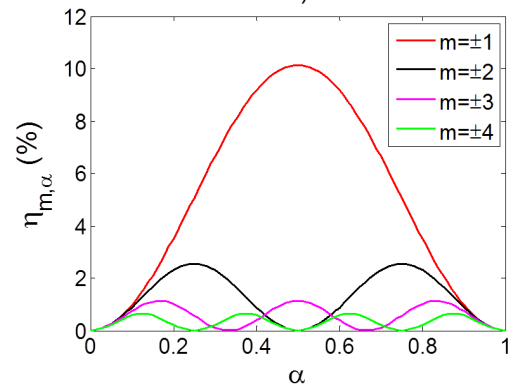

Fig. 6. Diffraction efficiency of the amplitude mask apodized with variable fill factor.

The power spectrum was determined for the amplitude uniform mask (with coefficients $a=1, b=0$ and constant $\alpha=0.5$ ) as well as for varying value of the fill factor (constant $\alpha=0.0625,0.125, \ldots, 0.5$ ), which is a kind of apodization, proceeding analogously as in the case of phase masks. Resulting power spectra are shown in Fig. 7.

a)

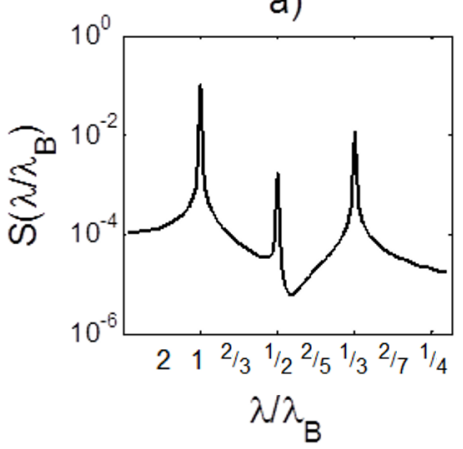

b)

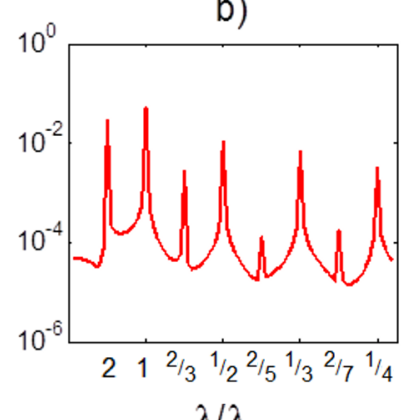

c)

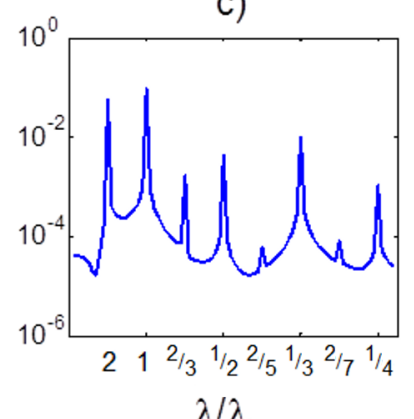

Fig. 7. Power spectra of the amplitude mask: a) uniform $(\alpha=0.5)$, b) with Gaussian apodization function and c) with Tanh apodization function, obtained by varying the fill factor.

Comparing the results for different apodization functions one can come to similar conclusions as in the case of phase masks. Namely, the power spectrum of grating with Tanh apodization is more similar to the power spectrum of the FBG written using the uniform mask. Moreover, for Gaussian and Tanh apodization profiles both power spectra consist of all harmonic components. This is due to the fact, that in the case of apodized amplitude masks, all existing (i.e. $m=0$ and $m= \pm 1, \ldots, \pm 4$ ) diffraction orders are non-zero.

Next, with reference to results obtained for apodized phase masks (Fig. 4) it can be seen that the values of peak amplitudes are significantly smaller. This is due to the fact, that the amplitude mask is only partly transparent $(b=0)$. Simultaneously, the relationships between particular peak amplitudes of the power spectra are the same as in the case of the mentioned phase masks. And so, the biggest values of peak amplitudes in the case of gratings with Tanh apodization are observed for harmonic $\lambda_{B}, \lambda_{B} / 2$ and $\lambda_{B} / 3$, whereas in the case of Gaussian apodization for spectral components corresponding to $2 \lambda_{B}, \lambda_{B}, \lambda_{B} / 2, \lambda_{B} / 3$, and $\lambda_{B} / 4$. An exception is harmonic $2 \lambda_{B}$, which is more dominant (especially for Tanh apodization) in the case of the amplitude mask comparing to the phase one. The reason is that diffraction efficiency of zeroth order has different character than in the case of phase mask. Therefore, it gives a greater contribution to the formation of the reflectance of the wavelength $2 \lambda_{B}$ (as a consequence of beating between $\mathrm{m}=0$ and $\mathrm{m}= \pm 1$ 
orders). Hence, the apodized amplitude masks can be considered as an alternative for the phase ones. However, due to their partial transparency, they may not be as effective as phase masks when used for gratings inscription. Moreover, gratings obtained with this method do not possess constant effective refraction index along its length and therefore they can be applied only if reflectance spectrum broadening for shorter wavelengths does not excludes their use.

\section{Conclusions}

The harmonic responses of apodized FBGs written using phase masks with variable phase step height and variable fill factor were analyzed numerically. Additionally, similar simulations for the apodized amplitude mask were made. These results show that both the methods of apodization (variable $\phi$ or $\alpha$ ) and the shape of the apodizing function have a significant impact on the power spectra of the resulting apodized FBGs. Thus, an appropriate selection of the apodization method and the apodization profile allow obtaining complex power spectra and optimize mutual relation between reflection efficiencies for Bragg wavelength and its harmonics. This knowledge can be used in practice during the process of FBG exposure (optimized for operation at the harmonics of Bragg wavelength) and in sensor applications where the simultaneous monitoring of the Bragg wavelength and its harmonic is possible.

\section{Acknowledgements}

The research for this paper was financially supported by the statutory work of the National Institute of Telecommunications for the 2016 entitled "Theoretical and experimental studies in the field of photonic structures for metrology, sensor technology, optics and fiber telecommunications". 